\title{
Density of Chromatic Roots in Minor-Closed Graph Families
}

\section{Perrett, Thomas J.; Thomassen, Carsten}

\section{Published in:}

Combinatorics, Probability \& Computing

Link to article, DOI:

$10.1017 /$ S0963548318000184

Publication date:

2018

Document Version

Peer reviewed version

Link back to DTU Orbit

Citation (APA):

Perrett, T. J., \& Thomassen, C. (2018). Density of Chromatic Roots in Minor-Closed Graph Families.

Combinatorics, Probability \& Computing, 27(6), 988-998. https://doi.org/10.1017/S0963548318000184

\section{General rights}

Copyright and moral rights for the publications made accessible in the public portal are retained by the authors and/or other copyright owners and it is a condition of accessing publications that users recognise and abide by the legal requirements associated with these rights.

- Users may download and print one copy of any publication from the public portal for the purpose of private study or research.

- You may not further distribute the material or use it for any profit-making activity or commercial gain

- You may freely distribute the URL identifying the publication in the public portal

If you believe that this document breaches copyright please contact us providing details, and we will remove access to the work immediately and investigate your claim. 


\title{
Density of chromatic roots in minor-closed graph families
}

\author{
Thomas J. Perrett* and Carsten Thomassen* \\ Department of Applied Mathematics and Computer Science, \\ Technical University of Denmark, DK-2800 Lyngby, Denmark.
}

February 19, 2018

\begin{abstract}
We prove that the roots of the chromatic polynomials of planar graphs are dense in the interval between $32 / 27$ and 4, except possibly in a small interval around $\tau+2$ where $\tau$ is the golden ratio. This interval arises due to a classical result of Tutte, which states that the chromatic polynomial of every planar graph takes a positive value at $\tau+2$. Our results lead us to conjecture that $\tau+2$ is the only such number less than 4 .
\end{abstract}

Keywords: roots of chromatic polynomials, minor-closed graph families. $\operatorname{MSC}(2010): 05 \mathrm{C} 15,05 \mathrm{C} 31,05 \mathrm{C} 83$.

\section{Introduction}

The chromatic polynomial was introduced in 1912 by Birkhoff [1] in order to study the 4colour problem which says that 4 is not a root of the chromatic polynomial of any planar graph. Since then, chromatic polynomials and their roots, which we call chromatic roots, have also become of interest in statistical physics, see e.g. [7], where complex chromatic roots are studied. In the present paper we deal exclusively with real chromatic roots. If $\mathcal{G}$ is a class of graphs, then we let $R(\mathcal{G})$ denote the set of real chromatic roots of the graphs in $\mathcal{G}$.

It is easy to see that 0 and 1 are the smallest chromatic roots. Jackson [3] proved the surprising result that every other chromatic root is strictly greater than $32 / 27$ and that this bound is sharp. Thomassen [8] proved that chromatic roots are dense in the interval from $32 / 27$ to infinity, so that the chromatic roots of all graphs are understood in the sense that if $\mathcal{G}$ denotes the class of all graphs, then $\overline{R(\mathcal{G})}=\{0,1\} \cup[32 / 27, \infty)$. Here, $\bar{A}$ denotes the closure of a set $A$.

By contrast, the situation for planar graphs is much less clear. Birkhoff and Lewis [2] proved that all chromatic roots of planar graphs are less than 5 , and conjectured that the

\footnotetext{
${ }^{*}$ Research supported by ERC Advanced Grant GRACOL, project number 320812.
} 
same should hold with 5 replaced by 4 . On the other hand, Thomassen [8] proved that the chromatic roots of planar graphs are dense in the interval from $32 / 27$ to 3 . Thus, for planar graphs, the remaining interval from 3 to 4 is of interest.

Conjecture 1.1. [8] If $\mathcal{G}$ denotes the family of planar graphs, then $[3,4] \subseteq \overline{R(\mathcal{G})}$.

Recently, Royle [4] proved that real chromatic roots of planar triangulations can be found in any interval $(4-\varepsilon, 4)$ for $\varepsilon>0$. In the present paper we prove that the chromatic roots of planar graphs are dense in most of the interval $[3,4]$, almost settling Conjecture 1.1.

Theorem 1.2. The set of chromatic roots of planar graphs contains a dense subset of $\left(3, t_{1}\right) \cup$ $\left(t_{2}, 4\right)$, where $t_{1}$ and $t_{2}$ are constants with $t_{1} \approx 3.618032$ and $t_{2} \approx 3.618356$.

To prove Theorem 1.2, we develop a technique which relates the set $R(\mathcal{G})$ to the numbers at which the chromatic polynomial of some graph in $\mathcal{G}$ is negative. The unsolved interval $\left(t_{1}, t_{2}\right)$ in Theorem 1.2 arises because our technique breaks down in connection with the following fascinating result of Tutte.

Theorem 1.3. [9] If $G$ is a planar triangulation, then $P(G, \tau+2)>0$, where $\tau$ is the golden ratio.

Indeed, note that $\tau+2 \approx 3.618033$ which falls within the interval $\left(t_{1}, t_{2}\right)$. Thus it is possible that Tutte's result [9] can be extended to a small neighbourhood around $\tau+2$. However, we do not believe this to be the case. In fact, the intermediate results in this paper lead us to a conjecture that $\tau+2$ is the only number in the interval $(3,4)$ at which the chromatic polynomials of planar graphs are always positive.

Finally, we remark that one can ask similar questions about the set $R(\mathcal{G})$ for other classes of graphs $\mathcal{G}$. In [5], the techniques of this paper are used to prove results regarding the classes of $K_{3,3}$-minor free graphs, $K_{5}$-minor free graphs, and graphs on surfaces. Indeed, when $\mathcal{G}$ denotes the $K_{3,3}$-minor free graphs, we provide a complete characterisation of $R(\mathcal{G})$ unless there are counterexamples to the Birkhoff and Lewis conjecture.

\section{Chromatic roots of minor-closed graph families}

Let $\mathcal{G}$ be a possibly infinite family of finite graphs. Recall that $R(\mathcal{G})$ denotes the set of all real chromatic roots of graphs in $\mathcal{G}$. Similarly, we denote by $R_{o}(\mathcal{G})$ the set of all chromatic roots of odd multiplicity of graphs in $\mathcal{G}$. Finally, we denote by $N(\mathcal{G})$ the set of all real numbers $q$ such that the chromatic polynomial of some graph in $\mathcal{G}$ is negative at $q$. It is easy to show that these sets are related.

Lemma 2.1. If $\mathcal{G}$ is a class of graphs, then $R_{o}(\mathcal{G}) \subseteq \overline{N(\mathcal{G})}$. 
Proof. Let $q_{0}$ be a real number such that $q_{0} \in R_{o}(\mathcal{G})$. Thus, for some graph $G$ in $\mathcal{G}$, the chromatic polynomial $P(G, q)$ is of the form $\left(q-q_{0}\right)^{r} S(q)$ where $r$ is an odd natural number and $S$ is a polynomial such that $S\left(q_{0}\right) \neq 0$. By continuity, $S(q)$ is non-zero in an interval $\left(q_{0}-\varepsilon, q_{0}+\varepsilon\right)$ for some $\varepsilon>0$. Now, for any $\varepsilon^{\prime}$ such that $0<\varepsilon^{\prime}<\varepsilon$, it follows that precisely one of $P\left(G, q_{0}-\varepsilon^{\prime}\right)$ and $P\left(G, q_{0}+\varepsilon^{\prime}\right)$ is negative. Thus, $q_{0}$ is in the closure of $N(\mathcal{G})$.

For two graphs $G$ and $H$, and an integer $k \geq 2$, a $k$-clique sum of $G$ and $H$ is any graph formed by identifying a clique of size $k$ in $G$ with a clique of size $k$ in $H$. Thomassen [8] showed that in certain circumstances, a construction based on a 2-clique sum can produce a graph with a chromatic root in a given interval.

Proposition 2.2. [8] Let $q_{0}$ and $\delta$ be real numbers with $q_{0}>2$ and $\delta>0$. Let $G$ be $a$ graph, and $e \in E(G)$. Let $H_{k, \ell}^{\prime}$ be the graph formed by a 2-clique sum of $k$ copies of $G$, and $\ell$ copies of $C_{4}$, where the edge identified is e. Finally, let $H_{k, \ell}$ be the graph formed from $H_{k, \ell}^{\prime}$ by deleting the edge corresponding to e. If $\left|P\left(G, q_{0}\right)\right|<\left(q_{0}-1\right)\left|P\left(G / e, q_{0}\right)\right|$ and $P\left(G, q_{0}\right) P\left(G / e, q_{0}\right)<0$, then there exist $k, \ell \in \mathbb{N}$, and a real number $q_{1} \in\left(q_{0}-\delta, q_{0}+\delta\right)$ such that $q_{1} \in R_{o}\left(\left\{H_{k, \ell}\right\}\right)$.

We say that $\mathcal{G}$ is closed under taking $k$-clique sums if all $k$-clique sums of graphs $G, H \in \mathcal{G}$ are members of $\mathcal{G}$. Under certain conditions, we can now prove a stronger relationship between the closures of the sets $R_{o}(\mathcal{G})$ and $N(\mathcal{G})$.

Theorem 2.3. Let $\mathcal{G}$ be a class of graphs such that $C_{4} \in \mathcal{G}$. If $\mathcal{G}$ is closed under edge deletion and taking 2 -clique sums, then $\overline{R_{o}(\mathcal{G})} \cap[2, \infty)=\overline{N(\mathcal{G})} \cap[2, \infty)$.

Proof of Theorem 2.3. By Lemma 2.1, it suffices to show that $N(\mathcal{G}) \cap[2, \infty) \subseteq \overline{R_{o}(\mathcal{G})}$, so suppose that $q_{0} \in N(\mathcal{G}) \cap[2, \infty)$. Thus, there is a graph $G \in \mathcal{G}$, such that $P\left(G, q_{0}\right)<0$. This implies that $q_{0} \neq 2$, because the chromatic polynomial of any graph evaluated at a positive integer is non-negative. Assume that $G$ is edge-minimal with this property, so for each edge $e$ we have $P\left(G-e, q_{0}\right) \geq 0$. Note that $G$ is not edgeless, since the chromatic polynomial of a graph with no edges is positive at any $q_{0}>0$. By the deletion-contraction formula, we have

$$
P\left(G-e, q_{0}\right)=P\left(G, q_{0}\right)+P\left(G / e, q_{0}\right) .
$$

Hence $P\left(G / e, q_{0}\right) \geq-P\left(G, q_{0}\right)$ and $P\left(G / e, q_{0}\right)>0$, and so $G$ and $q_{0}$ satisfy the conditions in Proposition 2.2, which implies that for all $\delta>0$, there is a graph $H$ such that $P(H, q)$ has a root of odd multiplicity in $\left(q_{0}-\delta, q_{0}+\delta\right)$. Furthermore, $H \in \mathcal{G}$ since $C_{4} \in \mathcal{G}$ and $\mathcal{G}$ is closed under edge deletion and taking 2 -clique sums. Thus $q_{0} \in \overline{R_{o}(\mathcal{G})}$.

Let $\mathcal{G}$ be a minor-closed class of graphs, and denote by $\operatorname{Forb}(\mathcal{G})$ the minor-minimal graphs not in $\mathcal{G}$. We note the following simple fact. 
Proposition 2.4. If $\mathcal{G}$ is a minor-closed class of graphs such that all graphs in Forb $(\mathcal{G})$ are 3 -connected, then $\mathcal{G}$ is closed under taking 2 -clique sums.

Proof. Let $G$ be a graph formed by a 2 -clique sum of graphs $G_{1}, G_{2} \in \mathcal{G}$. If $G$ contains a 3-connected minor $F$, then $F$ must be a minor of $G_{1}$ or $G_{2}$, a contradiction. Hence $G \in \mathcal{G}$ as required.

Since minor-closed classes of graphs are clearly closed under edge-deletion, we deduce the following from Theorem 2.3 and Proposition 2.4.

Theorem 2.5. Let $\mathcal{G}$ be a minor-closed class of graphs such that $C_{4} \in \mathcal{G}$. If all graphs in Forb $(\mathcal{G})$ are 3 -connected, then $\overline{R_{o}(\mathcal{G})} \cap[2, \infty)=\overline{N(\mathcal{G})} \cap[2, \infty)$.

We note one final relationship, which is a simple consequence of the addition-contraction formula. If $\mathcal{G}$ is a class of graphs, let $\mathcal{G}_{\max }$ denote the set of edge-maximal graphs in $\mathcal{G}$. Also, let $A(\mathcal{G})$ denote the set of real numbers such that $P(G, q)>0$ for every $G \in \mathcal{G}$.

Lemma 2.6. If $\mathcal{G}$ is a minor-closed class of graphs, then $A(\mathcal{G})=A\left(\mathcal{G}_{\max }\right)$.

Proof. Clearly $A(\mathcal{G}) \subseteq A\left(\mathcal{G}_{\max }\right)$ hence it just remains to prove the reverse inclusion. To this end, suppose for a contradiction that there is $q \in A\left(\mathcal{G}_{\max }\right)$ such that $q \notin A(\mathcal{G})$. Thus, there is a graph $G \in \mathcal{G}$ such that $P(G, q) \leq 0$. Suppose that $G$ has as few vertices as possible

and, subject to that, suppose further that $G$ is edge maximal. Since $q \in A\left(\mathcal{G}_{\max }\right)$, we have that $G$ is not edge-maximal. Thus there are $u, v \in V(G)$ such that $G+u v \in \mathcal{G}$. Now by the addition-contraction formula, we have

$$
P(G, q)=P(G+u v, q)+P(G / u v, q) .
$$

Since $\mathcal{G}$ is minor-closed, we also have that $G / u v \in \mathcal{G}$. Furthermore, by the vertex-minimality and edge-maximality assumptions on $G$, we have $P(G+u v, q)>0$ and $P(G / u v, q)>0$. It follows from (1) that $P(G, q)>0$, a contradiction.

\section{Chromatic roots of planar graphs}

In this section we apply Theorem 2.5 to deduce Theorem 1.2. However we first discuss the current situation with regards to the chromatic roots of the set $\mathcal{G}$ of planar graphs. Recall the result of Jackson mentioned in the introduction and the following widely believed conjecture of Birkhoff and Lewis.

Conjecture 3.1. [2] A planar graph has no chromatic root in the interval $[4, \infty)$. 


\begin{tabular}{rrr}
\hline & $p_{1}$ & $p_{2}$ \\
\hline$q^{16}$ & 1 & \\
$q^{15}$ & -48 & 1 \\
$q^{14}$ & 1098 & -45 \\
$q^{13}$ & -15902 & 961 \\
$q^{12}$ & 163358 & -12934 \\
$q^{11}$ & -1263582 & 122848 \\
$q^{10}$ & 7622430 & -873498 \\
$q^{9}$ & -36631145 & 4811140 \\
$q^{8}$ & 141942413 & -20939539 \\
$q^{7}$ & -445623415 & 72739711 \\
$q^{6}$ & 1131241573 & -202024440 \\
$q^{5}$ & -2299985643 & 445549685 \\
$q^{4}$ & 3673360947 & -766835653 \\
$q^{3}$ & -4454394269 & 996884682 \\
$q^{2}$ & 3863934525 & -923047942 \\
$q^{1}$ & -2138431325 & 543148340 \\
$q^{0}$ & 567438039 & -152643002 \\
\hline
\end{tabular}

Figure 1: Coefficients of the polynomials $p_{1}$ and $p_{2}$.

Collectively, these would imply that $R(\mathcal{G}) \subseteq\{0,1\} \cup(32 / 27,4)$. Moreover, the result of Thomassen and the work of this paper suggests that $\overline{R(\mathcal{G})}=\{0,1\} \cup[32 / 27,4]$. However this simple statement does not capture the richness of behaviour exhibited by the chromatic polynomials of planar graphs.

Indeed, recall Theorem 1.3 which says that $\tau+2 \in A\left(\mathcal{G}_{\max }\right)$ where $\mathcal{G}$ is the set of planar graphs. Applying Lemma 2.6, one may deduce that Theorem 1.3, holds not just for planar triangulations, but for all planar graphs. We suspect this is known, though we include it here as we have been unable to find a written reference.

Theorem 3.2. If $\mathcal{G}$ denotes the class of planar graphs, then $\tau+2 \in A(\mathcal{G})$.

It follows naturally to ask for a precise description of the set $A(\mathcal{G})$ where $\mathcal{G}$ denotes the class of planar graphs. We believe the answer to be the following.

Conjecture 3.3. If $\mathcal{G}$ denotes the class of planar graphs, then $A(\mathcal{G})=\{\tau+2\} \cup[4, \infty)$.

Note that Conjecture 3.3 contains Conjecture 3.1, Theorem 3.2 and the Four-Colour Theorem. In addition, it also implies Conjecture 1.1 via an application of Theorem 2.5. In what follows we shall show that $\left(3, t_{1}\right) \cup\left(t_{2}, 4\right) \subseteq N(\mathcal{G})$, where $t_{1}$ and $t_{2}$ are defined below. Therefore, as in the problem of determining $R(\mathcal{G})$, the only part of Conjecture 3.3 that remains to be proven are the intervals $\left(t_{1}, t_{2}\right)$ and $(4,5)$. 

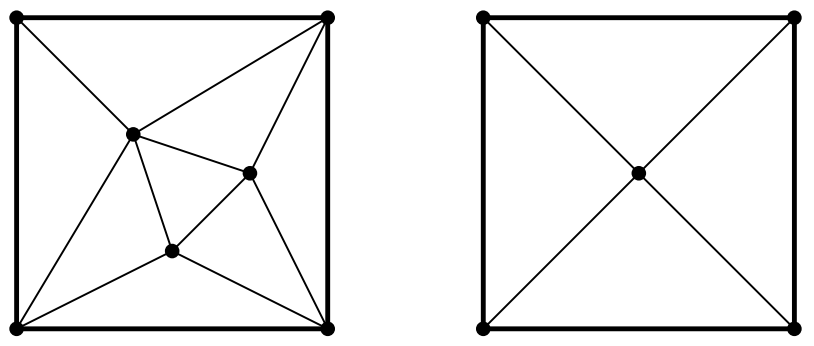

Figure 2: The graphs $F$ and $W$ with distinguished 4-cycles in bold.

Let $p_{1}$ and $p_{2}$ be the polynomials whose coefficients are listed in Figure 1 . Let $t_{1} \approx$ 3.618032 be the largest real root of $p_{1}$, and let $t_{2} \approx 3.618356$ be the second largest real root of $p_{2}$.

Lemma 3.4. If $G$ denotes the class of planar graphs, then $\left(3, t_{1}\right) \cup\left(t_{2}, 3.8\right) \subseteq N(\mathcal{G})$.

Proof. Let $F$ and $W$ be the graphs in Figure 2, and let $G_{1}$ be the unique graph obtained from the union of $F$ and $W$ by identifying the two distinguished 4-cycles. It may be calculated that $G_{1}$ has chromatic polynomial

$$
P\left(G_{1}, q\right)=q\left(q^{7}-18 q^{6}+141 q^{5}-619 q^{4}+1627 q^{3}-2525 q^{2}+2107 q-714\right),
$$

which is negative in the interval $(3,3.6)$.

Now let $K$ be the graph in Figure 3. Let $G_{2}$ denote the planar triangulation formed from $K$ and two copies of $F$, say $F_{1}$ and $F_{2}$, by identifying the two distinguished 4-cycles in $K$ with the distinguished 4-cycles in $F_{1}$ and $F_{2}$ respectively. We do this in such a way that the vertices of degree 3 in $F_{1}$ and $F_{2}$ are at distance 2 in $G_{2}$. It may be computed that this graph has chromatic polynomial

$$
P\left(G_{2}, q\right)=q(q-1)(q-2)(q-3)^{3} p_{1}(q)
$$

where $p_{1}$ is the polynomial in Figure 1 . Among other places, the polynomial $P\left(G_{2}, t\right)$ is negative in the interval $\left(3.5, t_{1}\right)$.

Finally, let $G_{3}$ be the unique planar triangulation formed from the disjoint union of $F, K$ and $W$ by identifying the two distinguished 4-cycles in $K$ with those in $F$ and $W$ respectively. It may be computed that the chromatic polynomial of this graph is

$$
P\left(G_{3}, q\right)=q(q-1)(q-2)(q-3)^{2} p_{2}(q)
$$

where $p_{2}$ is the polynomial in Figure 1 . The polynomial $P\left(G_{3}, q\right)$ is negative in the interval $\left(t_{2}, 3.8\right)$. 

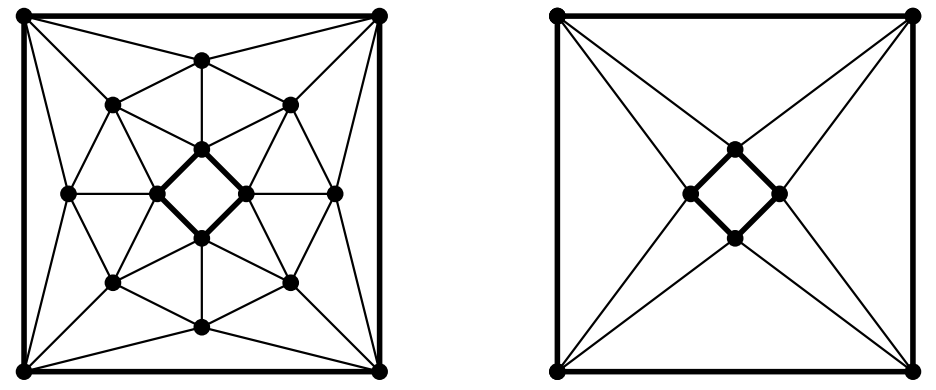

Figure 3: The graphs $K$ and $L$ with distinguished 4-cycles in bold.

In Section 4, we investigate an infinite family of planar triangulations $T(n), n \in \mathbb{N}$. These graphs are formed from the graphs $W, K$ and $L$ depicted in Figure 2 and Figure 3. We analyse their chromatic polynomials using a transfer matrix approach to obtain the following result.

Lemma 3.5. For every $q \in(3.7,4)$, there exists $n \in \mathbb{N}$ such that $P(T(n), q)<0$.

We are now able to prove Theorem 1.2.

Proof of Theorem 1.2. Let $\mathcal{G}$ denote the class of planar graphs. Lemma 3.4 and Lemma 3.5 show that $\left(3, t_{1}\right) \cup\left(t_{2}, 4\right) \subseteq N(\mathcal{G})$. By Wagner's Theorem [10], which is equivalent to Kuratowski's Theorem, $\operatorname{Forb}(\mathcal{G})=\left\{K_{5}, K_{3,3}\right\}$. Since both $K_{5}$ and $K_{3,3}$ are 3 -connected, Theorem 2.5 now implies the result.

\section{Proof of Lemma 3.5}

Let $K$ and $L$ be the graphs in Figure 3. We denote by $L^{n}$ the graph obtained from $n$ copies of $L$, say $L_{1}, \ldots, L_{n}$, by identifying the inner 4-cycle of $L_{i}$ with the outer 4-cycle of $L_{i+1}$ for each $i \in\{1, \ldots, n-1\}$. The resulting graph is planar and has two distinguished 4-cycles. Also let $W$ denote the wheel on 5 vertices, see Figure 2, and let $K^{\prime}$ denote the graph formed from $K$ by adding a single edge, triangulating one of the distinguished 4-cycles. In this section, we analyse the family of planar triangulations $T(n), n \in \mathbb{N}$, formed by identifying the distinguished 4-cycles of $L^{n}$ with the distinguished 4-cycles of $W$ and $K^{\prime}$ respectively.

The graphs $T(n), n \in \mathbb{N}$ were first studied by Royle [4], who took inspiration from a graph found by Woodall and from graphs studied in the field of statistical mechanics. Indeed, the family of graphs $L^{n}, n \in \mathbb{N}$ can be viewed as $4 \times n$ strips of the infinite triangular lattice, see [4, Figure 1], where one side of the strip is wrapped around and identified with the other. A standard technique to compute the chromatic polynomial of such graphs is the so-called transfer matrix approach, see [6]. We employ this technique in the form used by Royle [4], and give here a fairly condensed analysis. A more detailed presentation can be found in [4]. 


\begin{tabular}{crrrr}
\hline & $q_{1}$ & $q_{2}$ & $q_{3}$ & $q_{4}$ \\
\hline$q^{11}$ & & 1 & 1 & 1 \\
$q^{10}$ & & -34 & -34 & -34 \\
$q^{9}$ & 1 & 538 & 538 & 538 \\
$q^{8}$ & -28 & -5244 & -5244 & -5246 \\
$q^{7}$ & 362 & 35078 & 35078 & 35148 \\
$q^{6}$ & -2846 & -169490 & -169490 & -170548 \\
$q^{5}$ & 15036 & 604806 & 604806 & 613920 \\
$q^{4}$ & -55448 & -1595807 & -1595807 & -1645010 \\
$q^{3}$ & 142716 & 3051803 & 3051803 & 3222645 \\
$q^{2}$ & -246724 & -4024676 & -4024676 & -4397342 \\
$q^{1}$ & 258889 & 3286881 & 3286881 & 3753346 \\
$q^{0}$ & -124884 & -1255163 & -1255163 & -1511254 \\
\hline
\end{tabular}

Figure 4: Coefficients of the polynomials $q_{1}, q_{2}, q_{3}$ and $q_{4}$, see [4, Table 1].

Let $A$ be a graph with a distinguished 4-cycle $a_{1} a_{2} a_{3} a_{4}$. We may partition the colourings $\phi$ of $A$ into four types.

Type 1: $\phi\left(a_{1}\right)=\phi\left(a_{3}\right)$ and $\phi\left(a_{2}\right)=\phi\left(a_{4}\right)$,

Type 2: $\phi\left(a_{1}\right)=\phi\left(a_{3}\right)$ and $\phi\left(a_{2}\right) \neq \phi\left(a_{4}\right)$,

Type 3: $\phi\left(a_{1}\right) \neq \phi\left(a_{3}\right)$ and $\phi\left(a_{2}\right)=\phi\left(a_{4}\right)$,

Type 4: $\phi\left(a_{1}\right) \neq \phi\left(a_{3}\right)$ and $\phi\left(a_{2}\right) \neq \phi\left(a_{4}\right)$.

Let $P_{i}(A, q)$ denote the number of $q$-colourings of $A$ of type $i$. Note that identifying two vertices $a_{1}$ and $a_{3}$, say, gives a graph whose colourings correspond bijectively to the colourings $\phi$ of $A$ such that $\phi\left(a_{1}\right)=\phi\left(a_{3}\right)$. Alternatively, adding the edge $a_{1} a_{3}$ produces a graph whose colourings correspond bijectively to the colourings $\phi$ of $A$ such that $\phi\left(a_{1}\right) \neq \phi\left(a_{3}\right)$. Thus, for computational purposes, we have for example that $P_{2}(A, q)=P\left(A / a_{1} a_{3}+a_{2} a_{4}, q\right)$. We collect this information in a vector $Q(A, q)$ called the partitioned chromatic polynomial

$$
Q(A, q)=\left(\begin{array}{c}
P_{1}(A, q) \\
P_{2}(A, q) \\
P_{3}(A, q) \\
P_{4}(A, q)
\end{array}\right) .
$$

Let $\langle q\rangle_{k}$ denote the $k$ 'th falling factorial $q(q-1) \cdots(q-k+1)$ and let $q_{1}, \ldots, q_{4}$ be the polynomials whose coefficients are listed in Figure 4. The partitioned chromatic polynomials of $W$ and $K^{\prime}$ are given below. The formula for $Q(W, q)$ is trivial, whereas the formula for $Q\left(K^{\prime}, q\right)$ appears in [4] and was verified by the present authors. 


$$
Q(W, q)=\left(\begin{array}{c}
\langle q\rangle_{3} \\
\langle q\rangle_{4} \\
\langle q\rangle_{4} \\
\langle q\rangle_{5}
\end{array}\right) \quad Q\left(K^{\prime}, t\right)=\left(\begin{array}{c}
\langle q\rangle_{5} \cdot q_{1}(q) \\
\langle q\rangle_{4} \cdot q_{2}(q) \\
\langle q\rangle_{4} \cdot q_{3}(q) \\
\langle q\rangle_{4} \cdot(q-3) \cdot q_{4}(q)
\end{array}\right)
$$

In a similar way, for a graph with two distinguished 4-cycles, we may define a square matrix whose entries capture the types of colourings on those two 4-cycles. More precisely, we let the element in the $i$ th row and $j$ th coloumn be the number of colourings of the graph which are of type $i$ on the outer distinguished 4-cycle and type $j$ on the inner distinguished 4-cycle. The matrix $M$ corresponding to the graph $L$ in Figure 3 is as follows. The expression appears in [4] and was verified by the present authors.

$$
M=\left(\begin{array}{cccc}
\langle q\rangle_{4} & \langle q\rangle_{5} & \langle q\rangle_{5} & \langle q\rangle_{6} \\
\langle q\rangle_{5} & \langle q\rangle_{4}+2\langle q\rangle_{5}+\langle q\rangle_{6} & \langle q\rangle_{4}+2\langle q\rangle_{5}+\langle q\rangle_{6} & 4\langle q\rangle_{5}+4\langle q\rangle_{6}+\langle q\rangle_{7} \\
\langle q\rangle_{5} & \langle q\rangle_{4}+2\langle q\rangle_{5}+\langle q\rangle_{6} & \langle q\rangle_{4}+2\langle q\rangle_{5}+\langle q\rangle_{6} & 4\langle q\rangle_{5}+4\langle q\rangle_{6}+\langle q\rangle_{7} \\
\langle q\rangle_{6} & 4\langle q\rangle_{5}+4\langle q\rangle_{6}+\langle q\rangle_{7} & 4\langle q\rangle_{5}+4\langle q\rangle_{6}+\langle q\rangle_{7} & M_{44}
\end{array}\right)
$$

where $M_{44}=2\langle q\rangle_{4}+16\langle q\rangle_{5}+20\langle q\rangle_{6}+8\langle q\rangle_{7}+\langle q\rangle_{8}$. We can now state a lemma of Royle.

Lemma 4.1. [4] Let $A$ and $B$ be graphs with distinguished 4-cycles. If $X_{A, B}(n)$ denotes the graph obtained from $L^{n}$ by identifying its two distinguished 4-cycles with those of $A$ and $B$ respectively, then the chromatic polynomial of $X_{A, B}(n)$ is the sole entry of the $1 \times 1$ matrix

$$
Q(A)^{T} D(M D)^{n} Q(B)
$$

where

$$
D=\left(\begin{array}{cccc}
1 /\langle q\rangle_{2} & 0 & 0 & 0 \\
0 & 1 /\langle q\rangle_{3} & 0 & 0 \\
0 & 0 & 1 /\langle q\rangle_{3} & 0 \\
0 & 0 & 0 & 1 /\langle q\rangle_{4}
\end{array}\right)
$$

The matrix $M D$ is called the transfer matrix of the graph $L$, as it describes how colourings transfer from one 4-cycle to the other. As we let $n$ tend to infinity, the matrix $(M D)^{n}$ is determined by the spectral properties of $M D$. Indeed let $\lambda_{1}, \ldots, \lambda_{4}$ and $v_{1}, \ldots, v_{4}$ denote the eigenvalues and eigenvectors of $M D$ respectively. Furthermore, let $\|v\|$ denote the norm of the vector $v$ with respect to the inner product $\langle u, v\rangle=u^{T} D v$. Royle proves the following.

Lemma 4.2. [4] If $A$ and $B$ are graphs with distinguished 4-cycles and $q$ is a fixed real number in $(\tau+2,4)$, then

$$
P\left(X_{A, B}(n), q\right)=\alpha_{1} \beta_{1} \lambda_{1}^{n}\left\|v_{1}\right\|^{2}+\alpha_{2} \beta_{2} \lambda_{2}^{n}\left\|v_{2}\right\|^{2}+\alpha_{3} \beta_{3} \lambda_{3}^{n}\left\|v_{3}\right\|^{2}+\alpha_{4} \beta_{4} \lambda_{4}^{n}\left\|v_{4}\right\|^{2},
$$


where $\alpha_{i}\left\|v_{i}\right\|^{2}=Q(A, q)^{T} D v_{i}$ and $\beta_{i}\left\|v_{i}\right\|^{2}=Q(B, q)^{T} D v_{i}$ for $i \in\{1,2,3,4\}$.

In essence, Lemma 4.2 says that if $\lambda_{i}>0$ is the eigenvalue of largest absolute value, then in the limit as $n$ tends to infinity, the chromatic polynomial of $X_{A, B}(n)$ has the sign of $\alpha_{i} \beta_{i}$. Moreover, this sign can be determined simply by looking at the partitioned chromatic polynomials of $A$ and $B$ and the eigenvector $v_{i}$. With this in mind we first find the eigenvalues and eigenvectors of $M D$. Indeed, if $a$ is the polynomial $q^{4}-10 q^{3}+43 q^{2}-106 q+129$, then we have that $\lambda_{1}=2$,

$$
\begin{aligned}
& \lambda_{2}=\frac{1}{2}(q-3)\left(q^{3}-9 q^{2}+33 q-48-(q-4) a(q)^{1 / 2}\right), \\
& \lambda_{3}=\frac{1}{2}(q-3)\left(q^{3}-9 q^{2}+33 q-48+(q-4) a(q)^{1 / 2}\right),
\end{aligned}
$$

and $\lambda_{4}=0$. The corresponding eigenvectors are $v_{1}=(1,-1,-1,1)^{T}$,

$$
\begin{aligned}
& v_{2}=\left(\begin{array}{c}
\left(q^{2}-7 q+15+a(q)^{1 / 2}\right) /\left(2(q-2)(q-3)^{2}\right) \\
\left(q^{2}-9 q+21+a(q)^{1 / 2}\right) /\left(4(q-3)^{2}\right) \\
\left(q^{2}-9 q+21+a(q)^{1 / 2}\right) /\left(4(q-3)^{2}\right) \\
-1
\end{array}\right), \\
& v_{3}=\left(\begin{array}{c}
\left(q^{2}-7 q+15-a(q)^{1 / 2}\right) /\left(2(q-2)(q-3)^{2}\right) \\
\left(q^{2}-9 q+21-a(q)^{1 / 2}\right) /\left(4(q-3)^{2}\right) \\
\left(q^{2}-9 q+21-a(q)^{1 / 2}\right) /\left(4(q-3)^{2}\right) \\
-1
\end{array}\right),
\end{aligned}
$$

and $v_{4}=(0,1,-1,0)^{T}$.

We remark that in the notation of this section, the graphs $T(n)$ in Lemma 3.5 can be denoted $X_{K^{\prime}, W}(n)$. We can now prove Lemma 3.5.

Proof of Lemma 3.5. Let $t$ be a fixed real number in $(3.7,4)$. At $t$, we have $\lambda_{1}>\lambda_{2}>$ $\lambda_{3}>\lambda_{4}=0$. For $i \in\{1,2,3,4\}$, let $\alpha_{i}$ be such that $\alpha_{i}\left\|v_{i}\right\|^{2}=Q\left(K^{\prime}, t\right)^{T} D v_{i}$, and let $\beta_{i}$ be the corresponding value for the graph $W$. It may be calculated that $\alpha_{1}\left\|v_{1}\right\|^{2}=0$ and $\beta_{1}\left\|v_{1}\right\|^{2}=0$. Similarly, if $b$ denotes the polynomial $q^{3}-9 q^{2}+25 q-24$, and the polynomials $c$ and $d$ are defined as in Figure 5, then it may be calculated that

$$
\alpha_{2}\left\|v_{2}\right\|^{2}=\frac{d(q) a(q)^{1 / 2}-c(q)}{2(q-3)},
$$

and

$$
\beta_{2}\left\|v_{2}\right\|^{2}=\frac{(q-2) a(q)^{1 / 2}-b(q)}{2(q-3)^{2}} .
$$


From these expressions and by Lemma 4.2, the dominant term in the expression for $P(T(n), q)$ is $\alpha_{2} \beta_{2} \lambda_{2}^{n}\left\|v_{2}\right\|^{2}$. Thus, for large enough $n$, the sign of $P(T(n), q)$ depends on the sign of $\alpha_{2} \beta_{2}$. A short calculation gives that $\alpha_{2}\left\|v_{2}\right\|^{2}<0$ for $q \in(3.7,4)$ and $\beta_{2}\left\|v_{2}\right\|^{2}>0$ for $q \in(3,4)$, which implies that $\alpha_{2} \beta_{2}$ is negative in $(3.7,4)$. Therefore, there is $n \in \mathbb{N}$ such that $P(T(n), q)<0$.

\begin{tabular}{lrr}
\hline & $c$ & $d$ \\
\hline$q^{13}$ & 1 & \\
$q^{12}$ & -38 & \\
$q^{11}$ & 676 & 1 \\
$q^{10}$ & -7473 & -33 \\
$q^{9}$ & 57452 & 506 \\
$q^{8}$ & -325572 & -4770 \\
$q^{7}$ & 1405168 & 30784 \\
$q^{6}$ & -4698525 & -143070 \\
$q^{5}$ & 12224002 & 489214 \\
$q^{4}$ & -24510275 & -1231299 \\
$q^{3}$ & 36830333 & 2234215 \\
$q^{2}$ & -39168919 & -2778891 \\
$q^{1}$ & 26277660 & 2126441 \\
$q^{0}$ & -8337189 & -755627 \\
\hline
\end{tabular}

Figure 5: Coefficients of the polynomials $c$ and $d$.

\subsection{Remarks on the computations}

The calculation of chromatic polynomials in this article was done with MAPLE. The calculation of eigenvalues and eigenvectors of the matrix $M D$ was performed with MATLAB and cross-checked with the series expansion expressions presented in [4].

\subsection{Concluding remarks}

Theorem 1.2 is close to a proof of Conjecture 1.1. Indeed the missing interval $\left(t_{1}, t_{2}\right)$ has length less than 0.0004. Because of Tutte's result [9], it will require an infinite family of planar graphs to shrink this interval to the point $\tau+2$. It seems conceivable that for some graph $S$, the family $X_{S, W}(n), n \in \mathbb{N}$ may be useful to dispose of the interval $\left(\tau+2, t_{2}\right)$. Indeed, by mirroring the proof of Lemma 3.5, one only needs to find a graph $S$, with a distinguished 4-cycle, such that $Q(S, t) D v_{2}<0$ for $q \in\left(\tau+2, t_{2}\right)$. Royle observed the 
striking fact that the eigenvalues $\lambda_{1}, \ldots, \lambda_{4}$ are not distinct for $q=\tau+2$. However, they are distinct for $q \in(3,4) \backslash\{\tau+2\}$. Thus it seems possible that the families $X_{S, W}(n), n \in \mathbb{N}$ may also be useful to dispose of the interval $\left(t_{1}, \tau+2\right)$.

\section{References}

[1] G.D. Birkhoff, A determinant formula for the number of ways of coloring a map, Ann. of Math. 12 (1912) 42-46.

[2] G.D. Birkhoff and D.C. Lewis, Chromatic polynomials, Trans. Amer. Math. Soc. 60 (1946) 355-451.

[3] B. Jackson, A zero-free interval for chromatic polynomials of graphs. Combin. Probab. Comput. 2 (1993) 325-336.

[4] G. Royle, Planar triangulations with real chromatic roots arbitrarily close to four, Annals Combin. 12 (2008) 195-210.

[5] T.J. Perrett, Roots of the Chromatic Polynomial, Ph.D. Thesis, Technical University of Denmark, 2016.

[6] J. Salas and A.D. Sokal, Transfer Matrices and Partition-Function Zeros for Antiferromagnetic Potts Models. I. General Theory and Square-Lattice Chromatic Polynomial, J. Stat. Phys. 104 (2001) 609-699.

[7] A.D. Sokal, Chromatic roots are dense in the whole complex plane, Combin. Probab. Comput. 13 (2004) 221-261.

[8] C. Thomassen, The zero-free intervals for chromatic polynomials of graphs, Combin. Probab. Comput. 6 (1997) 497-506.

[9] W.T. Tutte, The golden ratio in the theory of chromatic polynomials, Ann. New York Acad. Sci. 175 (1970) 391-402.

[10] K. Wagner, Über eine Eigenschaft der ebenen Komplexe, Math. Ann. 114 (1937) 570590. 